\title{
Exponential stability of boundary controlled port Hamiltonian systems with dynamic feedback
}

\author{
Hector Ramirez * Hans Zwart ${ }^{* *}$ Yann Le Gorrec* \\ * Department of Automation and Micro-Mechatronic Systems, \\ FEMTO-ST UMR CNRS 6174, ENSMM, 26 chemin de l'épitaphe, \\ F-25030 Besançon, France. \{ramirez, legorrec\}@femto-st.fr \\ ** University of Twente, Faculty of Electrical Engineering, \\ Mathematics and Computer Science, Department of Applied \\ Mathematics, P.O. Box 2177500 AE Enschede, The Netherlands. \\ (e-mail:h.j.zwart@utwente.nl)
}

\begin{abstract}
In this paper it is shown that an input strictly passive linear finite dimensional port-Hamiltonian controller exponentially stabilizes a large class of boundary control systems. This follows since the finite dimensional controller dissipates the energy flowing through the boundaries of the infinite dimensional system. The assumptions on the controller is that it is input strictly passive and that it is exponentially stable. The result is illustrated on the model of a boundary controlled DNA-manipulation process.
\end{abstract}

Keywords: Boundary control systems, infinite dimensional port Hamiltonian systems, exponential stability, passivity.

\section{INTRODUCTION}

The study of stability and stabilization of infinite dimensional systems is a complicated task since extending results from the finite dimensional system theory is not straightforward and in many cases not possible. Concepts as dissipativity (Brogliato et al., 2007) have to be revised and well known results stated in the case of finite dimensional systems, as stability of interconnected dissipative systems, are no longer necessarily true in the infinite dimensional case. Boundary control systems (BCS) (Curtain and Zwart, 1995) are a class of abstract systems which model partial differential equations (PDEs) with the control and the observation at the boundary of the spatial domain. A large class of physical systems may be modelled as BCS, and very powerful results on well-posedness and stability have been reported for BCS formulated using the framework of infinite dimensional port-Hamiltonian system (Le Gorrec et al., 2005; Villegas, 2007; Villegas et al., 2009; Jacob and Zwart, 2012). More specifically in Villegas et al. (2009) it has been shown that a clever choice of the boundary conditions (by using a static feedback) renders the BCS exponentially stable, and in Villegas (2007); Ramirez and Le Gorrec (2013b) it has been shown that for a class of BCS arising from the modelling of physical systems, a power preserving interconnection with a finite dimensional passive linear system results in an asymptotically stable BCS on an extended state space. In this paper we show that the interconnection of a BCS with a linear finite dimensional controller renders the closedloop system exponentially stable provided that the finite

* This work was supported by French ANR sponsored project HAMECMOPSYS under Reference Code ANR-11-BS03-0002 dimensional system is input strictly passive and exponentially stable. This result permits to elegantly, and quite easily, prove the exponential stability for a large class of linear controllers, in particular those arising from energy shaping methods using Casimir functions (Macchelli and Melchiorri, 2004; Macchelli, 2012). The paper is organized as follows. In Section 2 we give the preliminaries on BCS. In section 3 we define the dynamic boundary controller and we derive a series of lemmas associated to the structure of the controller. In Section 4 we derive the main result of the paper, which is the exponential stability result of the BCS. Section 5 and Section 6 present the physical example of a DNA-manipulation process and the stabilizing control strategy. Section 7 presents some final remarks.

\section{BOUNDARY CONTROLLED PORT-HAMILTONIAN SYSTEMS}

The systems under study are described by the following PDE:

$$
\frac{\partial x}{\partial t}=P_{1} \frac{\partial}{\partial z}(\mathcal{L}(z) x(t, z))+\left(P_{0}-G_{0}\right) \mathcal{L}(z) x(t, z)
$$

where $z \in(a, b), P_{1} \in M_{n}(\mathbb{R})\left(M_{n}(\mathbb{R})\right.$ denotes the space of real $n \times n$ matrices) is a non-singular symmetric matrix, $P_{0}=-P_{0}^{\top} \in M_{n}(\mathbb{R}), G_{0} \geq 0 \in M_{n}(\mathbb{R})$ and $x$ takes values in $\mathbb{R}^{n}$. Furthermore, $\mathcal{L}(\cdot) \in L_{2}\left(a, b ; M_{n}(\mathbb{R})\right)$ is a bounded and continuously differentiable matrix-valued function satisfying for all $z \in(a, b), \mathcal{L}(z)=\mathcal{L}(z)^{\top}$ and $\mathcal{L}(z)>m I$, with $m$ independent from $z$. For simplicity $\mathcal{L}(z) x(t, z)$ will be denoted by $(\mathcal{L} x)(t, z)$. The state space is defined as $X=L_{2}\left(a, b ; \mathbb{R}^{n}\right)$ with inner product $\left\langle x_{1}, x_{2}\right\rangle_{\mathcal{L}}=$ $\left\langle x_{1}, \mathcal{L} x_{2}\right\rangle$ and norm $\|x\|_{\mathcal{L}}^{2}=\langle x, x\rangle_{\mathcal{L}}$. Hence $X$ is a Hilbert space. Note that the natural norm on $X$ and the $L_{2}$ norm 
are equivalent. The reason for selecting this space is that $\|\cdot\|_{\mathcal{L}}^{2}$ is usually related to the energy function of the system.

Definition 1. (Le Gorrec et al., 2005; Villegas et al., 2009) Let $\mathcal{L} x \in H^{1}\left(a, b ; \mathbb{R}^{n}\right)$. Then the boundary port variables associated with system (1) are the vectors $e_{\partial, \mathcal{L} x}, f_{\partial, \mathcal{L} x} \in$ $\mathbb{R}^{n}$, defined by

$$
\left[\begin{array}{c}
f_{\partial, \mathcal{L} x} \\
e_{\partial, \mathcal{L} x}
\end{array}\right]=\frac{1}{\sqrt{2}}\left[\begin{array}{cc}
P_{1} & -P_{1} \\
I & I
\end{array}\right]\left[\begin{array}{l}
(\mathcal{L} x)(b) \\
(\mathcal{L} x)(a)
\end{array}\right]=R\left[\begin{array}{l}
(\mathcal{L} x)(b) \\
(\mathcal{L} x)(a)
\end{array}\right]
$$

Note that the port variables are linear combinations of the boundary variables.

Theorem 2. (Villegas et al., 2009) Let $W$ be a $n \times 2 n$ real matrix. If $W$ has full rank and satisfies $W \Sigma W^{\top} \geq$ 0 , where $\Sigma=\left[\begin{array}{ll}0 & I \\ I & 0\end{array}\right]$, then the system (1), with input $u(t)=W\left[\begin{array}{l}f_{\partial, \mathcal{L} x}(t) \\ e_{\partial, \mathcal{L} x}(t)\end{array}\right]$ is a boundary control system on $X$. Furthermore, the operator $\mathcal{A} x=P_{1}(\partial / \partial z)(\mathcal{L} x)+\left(P_{0}-\right.$ $\left.G_{0}\right) \mathcal{L} x$ with domain

$$
D(\mathcal{A})=\left\{\mathcal{L} x \in H^{1}\left(a, b ; \mathbb{R}^{n}\right) \mid\left[\begin{array}{l}
f_{\partial, \mathcal{L} x} \\
e_{\partial, \mathcal{L} x}
\end{array}\right] \in \operatorname{ker} W\right\}
$$

generates a contraction semigroup on $X$. Let $\tilde{W}$ be a full rank matrix of size $n \times 2 n$ with $\left[\begin{array}{c}W \\ \tilde{W}\end{array}\right]$ invertible and let $P_{W, \tilde{W}}$ be given by

$$
P_{W, \tilde{W}}=\left(\left[\begin{array}{l}
W \\
\tilde{W}
\end{array}\right] \Sigma\left[\begin{array}{l}
W \\
\tilde{W}
\end{array}\right]^{\top}\right)^{-1}=\left[\begin{array}{ll}
W \Sigma W^{\top} & W \Sigma \tilde{W}^{\top} \\
\tilde{W} \Sigma W^{\top} & \tilde{W} \Sigma \tilde{W}^{\top}
\end{array}\right]^{-1} .
$$

Define the output of the system as the linear mapping $\mathcal{C}: \mathcal{L}^{-1} H^{1}\left(a, b ; \mathbb{R}^{n}\right) \rightarrow \mathbb{R}^{n}, y=\mathcal{C} x(t):=\tilde{W}\left[\begin{array}{l}f_{\partial, \mathcal{L} x}(t) \\ e_{\partial, \mathcal{L} x}(t)\end{array}\right]$. Then for $u \in C^{2}\left(0, \infty ; \mathbb{R}^{k}\right), \mathcal{L} x(0) \in H^{1}\left(a, b ; \mathbb{R}^{n}\right)$, and $u(0)=W\left[\begin{array}{l}f_{\partial, \mathcal{L}_{x}}(0) \\ e_{\partial, \mathcal{L} x}(0)\end{array}\right]$ the following balance equation is satisfied:

$$
\begin{aligned}
\frac{1}{2} \frac{d}{d t}\|x(t)\|_{\mathcal{L}}^{2} & =\frac{1}{2}\left[\begin{array}{l}
u(t) \\
y(t)
\end{array}\right]^{\top} P_{W, \tilde{W}}\left[\begin{array}{l}
u(t) \\
y(t)
\end{array}\right]-\left\langle G_{0} \mathcal{L} x(t), \mathcal{L} x(t)\right\rangle \\
& \leq \frac{1}{2}\left[\begin{array}{l}
u(t) \\
y(t)
\end{array}\right]^{\top} P_{W, \tilde{W}}\left[\begin{array}{l}
u(t) \\
y(t)
\end{array}\right] .
\end{aligned}
$$

The matrix $P_{W, \tilde{W}}$ is defined only when $\left[\begin{array}{c}W \\ \tilde{W}\end{array}\right]$ is invertible. Notice that in the absence of some internal dissipation $\left(G_{0}=0\right)$ the system only exchanges energy with the environment through the boundaries since the input and output act on the boundary of the spatial domain. Finally we remark that the balance equation (3) may be rewritten as:

$$
\frac{1}{2} \frac{d}{d t}\|x(t)\|_{\mathcal{L}}^{2} \leq\left[\begin{array}{l}
(\mathcal{L} x)(t, b) \\
(\mathcal{L} x)(t, a)
\end{array}\right]^{\top}\left[\begin{array}{cc}
P_{1} & 0 \\
0 & -P_{1}
\end{array}\right]\left[\begin{array}{l}
(\mathcal{L} x)(t, b) \\
(\mathcal{L} x)(t, a)
\end{array}\right]
$$

Remark 3. As it has been pointed out in Villegas (2007), if the matrices $W$ and $\tilde{W}$ are selected such that $P_{W, \tilde{W}}=$ $\left[\begin{array}{ll}0 & I \\ I & 0\end{array}\right]=\Sigma$, then the BCS fulfils $\frac{1}{2} \frac{d}{d t}\|x(t)\|_{\mathcal{L}}^{2} \leq u^{\top}(t) y(t)$.

In Villegas (2007) and Ramirez and Le Gorrec (2013b) it is shown that a power conserving interconnection (van der Schaft, 2000), i.e.,

$$
u=r-y_{c}, \quad y=u_{c},
$$

with $r \in \mathbb{R}^{n}$ the new input of the system, of a impedance energy preserving BCS, i.e., that satisfies $\frac{1}{2} \frac{d}{d t}\|x(t)\|_{\mathcal{L}}^{2}=$ $u(t)^{\top} y(t)$, and a linear strictly positive real (SPR) or strictly passive finite dimensional system defines again a BCS on an extended space. Consider the linear system

$$
\dot{v}=A_{c} v+B_{c} u_{c}, \quad y_{c}=C_{c} v+D_{c} u_{c},
$$

with state space $v \in V=\mathbb{R}^{m}$, set of input values $u_{c} \in U_{c}=\mathbb{R}^{n}$ and set of output values $y_{c} \in Y=\mathbb{R}^{n}$. The set $\mathcal{U}_{c}$ of admissible inputs consists of all $U_{c}$-valued piecewise continuous functions defined on $\mathbb{R}$. $A_{c}, B_{c}, C_{c}$ and $D_{c}$ are constant real matrices of dimension $m \times m$, $m \times n, n \times m$ and $n \times n$, respectively.

Theorem 4. (Villegas, 2007; Ramirez and Le Gorrec, $2013 \mathrm{~b})$ Let the state of the open-loop BCS satisfy $\frac{1}{2} \frac{d}{d t}\|x(t)\|_{\mathcal{L}}^{2}=u(t)^{\top} y(t)$. Consider a LTI strictly passive finite dimensional system with storage function $E_{c}(t)=$ $\frac{1}{2}\left\langle v(t), Q_{c} v(t)\right\rangle, Q_{c} \in M_{m}(\mathbb{R}), Q_{c}=Q_{c}^{\top}>0$. Then the feedback interconnection of the BCS and the finite dimensional system is again a BCS on the extended state space $\tilde{x} \in \tilde{X}=X \times V$ with inner product $\left\langle\tilde{x}_{1}, \tilde{x}_{2}\right\rangle_{\tilde{X}}=$ $\left\langle x_{1}, x_{2}\right\rangle_{\mathcal{L}}+\left\langle v_{1}, Q_{c} v_{2}\right\rangle$. Furthermore, the operator $\mathcal{A}_{e}$ defined by $\mathcal{A}_{e} \tilde{x}=\left[\begin{array}{cc}\mathcal{J} \mathcal{L} & 0 \\ B_{c} \mathcal{C} & A_{c}\end{array}\right]\left[\begin{array}{l}x \\ v\end{array}\right]$ with $D\left(\mathcal{A}_{e}\right)=\left\{\left[\begin{array}{l}x \\ v\end{array}\right] \in\right.$ $\left.\left[\begin{array}{l}X \\ V\end{array}\right] \mid \mathcal{L} x \in H^{N}\left(a, b ; \mathbb{R}^{n}\right),\left[\begin{array}{c}f_{\partial, \mathcal{L} x} \\ e_{\partial, \mathcal{L} x} \\ v\end{array}\right] \in \operatorname{ker} \tilde{W}_{D}\right\}$, where $\tilde{W}_{D}=\left[\left(W+D_{c} \tilde{W} C_{c}\right)\right]$ generates a contraction semigroup on $\tilde{X}$.

In this paper we show that not only asymptotic stability is assured Villegas (2007) if the controller is linear strictly passive, but also exponential stability.

Remark 5. For Theorem 4 to be fulfilled the matrices $W$ and $\tilde{W}$ should be selected such that $P_{W, \tilde{W}}=\left[\begin{array}{ll}0 & I \\ I & 0\end{array}\right]$.

Notice that the power preserving interconnection (5) actually defines a feedback loop, where the finite dimensional system acts as the controller.

\section{DYNAMIC BOUNDARY CONTROL}

The main result of this paper is proving that a strictly passive linear finite dimensional system exponentially stabilizes boundary controlled port Hamiltonian systems. This is a powerful result due to three reasons: 1) Exponential stability of infinite (and even finite) dimensional systems is a very strong condition difficult to prove. 2) A dynamic controller not only permits to stabilize the infinite dimensional system but also permits to change the closedloop equilibrium (using for instance Casimir methods). 3) In many applications the infinite dimensional system is coupled at the boundary with a finite dimensional system. In this case the finite dimensional system correspond to the "controller" and the stability of the coupled system may be analysed using our approach.

Definition 6. The considered finite dimensional controller is given by the state space representation:

$$
\dot{v}=\left(J_{c}-R_{c}\right) Q_{c} v+B_{c} u_{c}, \quad y_{c}=B_{c}^{\top} Q_{c} v+S_{c} u_{c},
$$
where we assume that $Q_{c}=Q_{c}^{\top}>0, J_{c}=-J_{c}^{\top}$, $R_{c}=R_{c}^{\top} \geq 0, S_{c}=S_{c}^{\top}>0$ and $B_{c}$ are real constant matrices of proper dimensions. Furthermore, the controller is assumed to be exponentially stable, i.e., $A_{c}:=\left(J_{c}-\right.$ $\left.R_{c}\right) Q_{c}$ is Hurwitz ${ }^{1}$.

\footnotetext{
1 This is equivalent to the pair $\left(J_{c}, R_{c}\right)$ being controllable
} 
Under the assumption made above, it is easy to see that with the Hamiltonian $E_{c}(t)=\frac{1}{2} v(t)^{\top} Q_{c} v(t)$ the system $(7)$ is a strictly input passive port-Hamiltonian system, i.e. there exists a $\sigma>0$ such that

$$
\dot{E}_{c}(t) \leq u_{c}(t)^{\top} y_{c}(t)-\sigma\left\|u_{c}(t)\right\|^{2} .
$$

We shall frequently use the following inequalities for $v, w \in$ $\mathbb{R}^{n}$ and $\alpha>0$

$$
\begin{aligned}
-\alpha^{2}\|v\|^{2}-\frac{1}{\alpha^{2}}\|w\|^{2} & \leq v^{\top} w+w^{\top} v \\
& \leq \alpha^{2}\|v\|^{2}+\frac{1}{\alpha^{2}}\|w\|^{2} .
\end{aligned}
$$

This holds since $\left\|\alpha v \pm \frac{1}{\alpha} w\right\|^{2} \geq 0$. The following lemmas follow from Definition 6 .

Lemma \%. There exist strictly positive constants $\kappa_{2}, \kappa_{3}$ and $\kappa_{4}$ such that for all $\tau>0$ the energy of (7) satisfies:

$$
E_{c}(\tau) \leq \kappa_{1}(\tau) E_{c}(0)+\kappa_{3} \int_{0}^{\tau}\left\|u_{c}(t)\right\|^{2} d t
$$

where $\kappa_{1}(\tau)=\kappa_{4} e^{-\kappa_{2} \tau}$.

Proof. Since $A_{c}=\left(J_{c}-R_{c}\right) Q_{c}$ is exponentially stable there exists $\kappa_{2}>0$ such that $A_{c}+2 \kappa_{2} I$ is still exponentially stable. Hence there exists a $P_{1}=P_{1}^{\top}>0$ satisfying

$$
\left(A_{c}+2 \kappa_{2} I\right)^{\top} P_{1}+P_{1}\left(A+2 \kappa_{2} I\right) \leq 0
$$

which implies that $A_{c}^{\top} P_{1}+P_{1} A_{c} \leq-4 \kappa_{2} P_{1}$. Taking the time derivative of $v^{\top} \stackrel{P}{P}_{1} v$ along trajectories we have

$$
\begin{aligned}
\frac{d}{d t} & \left(v^{\top} P_{1} v\right) \\
& =\left(A_{c} v+B_{c} u_{c}\right)^{\top} P_{1} v+v^{\top} P_{1}\left(A_{c} v+B_{c} u_{c}\right) \\
& \leq-4 \kappa_{2} v^{\top} P_{1} v+u_{c}^{\top} B_{c}^{\top} P_{1} v+v^{\top} P_{1} B_{c} u_{c} \\
& \leq-2 \kappa_{2} v^{\top} P_{1} v+\kappa_{3}\left\|u_{c}\right\|^{2}
\end{aligned}
$$

for some $\kappa_{3}>0$, where we used (9) with $\alpha^{2}=2 \kappa_{2}$. This implies that

$$
\frac{d}{d t}\left(e^{\kappa_{2} t} v^{\top}(t) P_{1} v(t)\right) \leq \kappa_{3} e^{\kappa_{2} t}\left\|u_{c}(t)\right\|^{2} .
$$

Integrating this relation over $t \in[0, \tau]$ and rearranging terms, we obtain $v(\tau)^{\top} P_{1} v(\tau) \leq e^{-\kappa_{2} \tau} v(0)^{\top} P_{1} v(0)+$ $\int_{0}^{\tau} \kappa_{3} e^{\kappa_{2}(t-\tau)}\left\|u_{c}(t)\right\|^{2} d t$. Since there exists positive constants $q_{1}, q_{2}$ such that for all $v \in \mathbb{R}^{n} q_{1} v^{\top} P_{1} v \leq \frac{1}{2} v^{\top} Q_{c} v \leq$ $q_{2} v^{\top} P_{1} v$, inequality (10) follows.

Lemma 8. There exists positive constants $\xi_{1}, \xi_{2}$ and $\tau_{0}$ such for all $\tau>\tau_{0}$ the energy of (7) satisfies

$\int_{0}^{\tau} E_{c}(t) d t \leq \xi_{1} \int_{0}^{\tau} v^{\top}(t) Q_{c} R_{c} Q_{c} v(t) d t+\xi_{2} \int_{0}^{\tau}\left\|u_{c}(t)\right\|^{2} d t$

Proof. Since $A_{c}=\left(J_{c}-R_{c}\right) Q_{c}$ is exponentially stable there exists a $P_{2}=P_{2}^{\top} \geq 0$ such that $A_{c}^{\top} P_{2}+P_{2} A_{c}=$ $-\frac{1}{2} Q_{c}$. Taking the time derivative of $v^{\top} P_{2} v$ we have

$$
\begin{aligned}
\frac{d}{d t} & \left(v^{\top} P_{2} v\right) \\
& =\left(A_{c} v+B_{c} u_{c}\right)^{\top} P_{2} v+v^{\top} P_{2}\left(A_{c} v+B_{c} u_{c}\right) \\
& =-\frac{1}{2} v^{\top} Q_{c} v+u_{c}^{\top} B_{c}^{\top} P_{2} v+v^{\top} P_{2} B_{c} u_{c} .
\end{aligned}
$$

Now, using equation (9) we find

$$
\frac{d}{d t}\left(v^{\top} P v\right) \leq\left(\alpha^{2}-1\right) \frac{1}{2} v^{\top} Q_{c} v+\frac{\beta_{3}}{\alpha^{2}} u_{c}^{\top} u_{c},
$$

for some $\beta_{3}>0$ and $\left.\alpha \in\right] 0,1[$. Now, integrating (15) we obtain

$$
\begin{aligned}
& \left(v^{\top} P v\right)(\tau)-\left(v^{\top} P_{2} v\right)(0) \leq \\
& \left(\alpha^{2}-1\right) \int_{0}^{\tau} E_{c}(t) d t+\frac{\beta_{3}}{\alpha^{2}} \int_{0}^{\tau}\left\|u_{c}(t)\right\|^{2} d t
\end{aligned}
$$

Since $P_{2}$ and $Q_{c}$ are symmetric and positive we may bound them by $P_{2} \leq \frac{1}{2} \beta_{5} Q_{c}$, with $\beta_{5}>0$ sufficiently large, to obtain

$$
\int_{0}^{\tau} E_{c}(t) d t \leq \beta_{1} E_{c}(0)+\beta_{2} \int_{0}^{\tau}\left\|u_{c}(t)\right\|^{2} d t .
$$

with $\beta_{1}=\frac{\beta_{5}}{\left(1-\alpha^{2}\right)}>0$ and $\beta_{2}=\frac{1}{\left(1-\alpha^{2}\right)} \frac{\beta_{3}}{\alpha^{2}}>0$.

On other hand we have that the time derivative of the energy of (7) is given by

$$
\begin{aligned}
& \frac{1}{2} \frac{d}{d t}\left(v^{\top} Q_{c} v\right)= \\
& \quad-v^{\top} Q_{c} R_{c} Q_{c} v+\frac{1}{2}\left(u_{c}^{\top} B_{c}^{\top} Q_{c} v+v^{\top} Q_{c} B_{c} u_{c}\right) .
\end{aligned}
$$

Using (8) in (18) we obtain we obtain for any $\eta>0$

$$
\begin{aligned}
& \frac{1}{2} \frac{d}{d t}\left(v^{\top} Q_{c} v\right) \geq \\
& \quad-v^{\top} Q_{c} R_{c} Q_{c} v-\frac{1}{2}\left(\eta^{2}\left\|Q_{c}^{\frac{1}{2}} B_{c} u_{c}\right\|^{2}+\frac{1}{\eta^{2}}\left\|Q_{c}^{\frac{1}{2}} v\right\|^{2}\right) .
\end{aligned}
$$

Integrating and grouping terms

$$
\begin{aligned}
& E_{c}(0) \leq E_{c}(\tau)+ \\
& \int_{0}^{\tau} v(t)^{\top} Q_{c} R_{c} Q_{c} v(t)+\frac{\eta^{2}}{2}\left\|Q_{c}^{\frac{1}{2}} B_{c} u_{c}(t)\right\|^{2}+\frac{1}{\eta^{2}} E_{c}(t) d t .
\end{aligned}
$$

Now, applying Lemma 7 and using (17) we obtain

$$
\begin{aligned}
E_{c}(0) \leq \kappa_{1}(\tau) E_{c}(0)+\kappa_{3} \int_{0}^{\tau}\left\|u_{c}(t)\right\|^{2} d t \\
+\int_{0}^{\tau} v^{\top} Q_{c} R_{c} Q_{c} v+\frac{\eta^{2}}{2}\left\|Q_{c}^{\frac{1}{2}} B_{c}\right\|^{2}\left\|u_{c}(t)\right\|^{2} d t \\
\quad+\frac{1}{\eta^{2}}\left(\beta_{1} E_{c}(0)+\beta_{2} \int_{0}^{\tau}\left\|u_{c}(t)\right\|^{2} d t\right) .
\end{aligned}
$$

Choosing $\tau_{0}$ and $\eta$ sufficiently large, such that $\kappa_{1}\left(\tau_{0}\right)+$ $\frac{\beta_{1}}{\eta^{2}}<1$, see Lemma 7 , we obtain for $\tau>\tau_{0}$

$$
E_{c}(0) \leq \gamma_{1} \int_{0}^{\tau} v(t)^{\top} Q_{c} R_{c} Q_{c} v(t) d t+\gamma_{2} \int_{0}^{\tau}\left\|u_{c}(t)\right\|^{2} d t
$$

with constants $\gamma_{1}=1 /\left(1-\left(\kappa_{1}\left(\tau_{0}\right)+\frac{\beta_{1}}{\eta^{2}}\right)\right)$ and $\gamma_{2}=$ $\left(\kappa_{3}+\frac{\eta^{2}}{2}\left\|Q_{c}^{\frac{1}{2}} B_{c}\right\|^{2}+\frac{\beta_{2}}{\eta^{2}}\right) \gamma_{1}$. Now, combining (17) and (19) we obtain

$$
\int_{0}^{\tau} E_{c}(t) d t \leq \xi_{1} \int_{0}^{\tau} v^{\top} Q_{c} R_{c} Q_{c} v d t+\xi_{2} \int_{0}^{\tau}\left\|u_{c}(t)\right\|^{2} d t
$$

with $\xi_{1}=\beta_{1} \gamma_{1}$ and $\xi_{2}=\beta_{1} \gamma_{2}+\beta_{2}$, which proves the Lemma.

Lemma 9. For every $\delta_{1}>0$ there exists a $\delta_{2}>0$ such that for all $\tau>0$ the energy of (7) satisfies the relation

$$
\int_{0}^{\tau} \delta_{1} E_{c}(t)+\left\|y_{c}(t)\right\|^{2} d t \leq \delta_{2} \int_{0}^{\tau} E_{c}(t)+\left\|u_{c}(t)\right\|^{2} d t .
$$

Proof. The relation follows by noting that the left term of (21) may be written and bounded as 


$$
\begin{array}{r}
\int_{0}^{\tau}\left[\begin{array}{c}
v \\
u_{c}
\end{array}\right]^{\top}\left[\begin{array}{rr}
\left(\frac{\delta_{1}}{2} Q_{c}+Q_{c} B_{c} B_{c}^{\top} Q_{c}\right) & Q_{c} B_{c} S_{c} \\
S_{c} B_{c}^{\top} Q_{c} & S_{c}^{2}
\end{array}\right]\left[\begin{array}{c}
v \\
u_{c}
\end{array}\right] d t \\
\leq \delta_{2} \int_{0}^{\tau}\left[\begin{array}{c}
v \\
u_{c}
\end{array}\right]^{\top}\left[\begin{array}{cc}
\frac{1}{2} Q_{c} & 0 \\
0 & I
\end{array}\right]\left[\begin{array}{c}
v \\
u_{c}
\end{array}\right] d t
\end{array}
$$

for some $\delta_{2}>0$.

\section{EXPONENTIAL STABILITY}

To show that the BCS defined in Theorem 4 is exponentially stable, we follow (Villegas et al., 2005; Villegas et al., 2009). In the rest of the paper, we will set $G_{0}=0$ for simplicity. Note, however, that all results are valid for $G_{0} \neq 0$. As a part of the boundary port variables of the infinite dimensional system can be set to zero and may be not used for the interconnection we will assume that the infinite dimensional system satisfies a dissipative relation. Assumption 10. The BCS of Theorem 2 satisfies

$$
\|u(t)\|^{2}+\|y(t)\|^{2} \geq \epsilon\|\mathcal{L} x(t, b)\|^{2}
$$

for some $\epsilon>0$.

To prove the main theorem we first present the following lemma which gives a bound on the total energy of the interconnected system.

Lemma 11. Consider a BCS as described in Theorem 4 with $r(t)=0$, for all $t \geq 0$. Then, the energy of the system $\tilde{E}(t)=\frac{1}{2}\|x(t)\|_{\mathcal{L}}^{2}+\frac{1}{2} v(t)^{T} Q_{c} v(t)$ satisfies for $\tau$ large enough

$$
\begin{aligned}
& \tilde{E}(\tau) \leq c(\tau) \int_{0}^{\tau}\|(\mathcal{L} x)(t, b)\|^{2} d t+\frac{2 c(\tau)}{c_{1}} \int_{0}^{\tau} E_{c}(t) d t \\
& \tilde{E}(\tau) \leq c(\tau) \int_{0}^{\tau}\|(\mathcal{L} x)(t, a)\|^{2} d t+\frac{2 c(\tau)}{c_{1}} \int_{0}^{\tau} E_{c}(t) d t
\end{aligned}
$$

where $c$ is a positive constant that only depends on $\tau$ and $c_{1}$ a positive constant.

Proof. In Villegas et al. (2009), it has been proved that there exist positive $\gamma$ and $\tau_{1}$ such that for $\tau>\tau_{1}>2 \gamma(b-$ a) the function $F(z)=\int_{\gamma(b-z)}^{\tau-\gamma(b-z)} x^{\top}(t, z) \mathcal{L}(z) x(t, z) d t$ fulfils $F(b) \geq F(z) e^{-\kappa(b-a)}$ for $z \in[a, b]$ where $\kappa$ is a positive constant. On other hand, due to the contraction property of the semigroup $\tilde{E}\left(t_{2}\right) \leq \tilde{E}\left(t_{1}\right)$ for $t_{2} \geq t_{1}$ it is deduced that

$$
\begin{aligned}
\int_{\gamma(b-z)}^{\tau-\gamma(b-z)} \tilde{E}(t) d t & \geq \tilde{E}(\tau-\gamma(b-a)) \int_{\gamma(b-z)}^{\tau-\gamma(b-z)} d t \\
& \geq(\tau-2 \gamma(b-a)) \tilde{E}(\tau-\gamma(b-a)) .
\end{aligned}
$$

Hence we obtain

$$
\begin{aligned}
2(\tau & -2 \gamma(b-a)) \tilde{E}(\tau) \\
& \leq 2(\tau-2 \gamma(b-a)) \tilde{E}(\tau-\gamma(b-a)) \\
& \leq \int_{a}^{b}\left(\int_{\gamma(b-z)}^{\tau-\gamma(b-z)} x^{\top}(\mathcal{L} x) d t\right) d z+2 \int_{\gamma(b-z)}^{\tau-\gamma(b-z)} E_{c} d t \\
& \leq \int_{a}^{b} F(z) d z+2 \int_{\gamma(b-z)}^{\tau-\gamma(b-z)} E_{c} d t \\
& \leq(b-a) F(b) e^{\kappa(b-a)}+2 \int_{\gamma(b-z)}^{\tau-\gamma(b-z)} E_{c} d t \\
& \leq c_{1} \int_{0}^{\tau}\|(\mathcal{L} x)(b)\|^{2} d t+2 \int_{\gamma(b-z)}^{\tau-\gamma(b-z)} E_{c} d t
\end{aligned}
$$

where $c_{1}=(b-a)\left\|\mathcal{L}^{-1}(b)\right\| e^{\kappa(b-a)}$. Hence we obtain that for $\tau>\tau_{1}, \tilde{E}(\tau) \leq c(\tau) \int_{0}^{\tau}\|(\mathcal{L} x)(t, b)\|^{2} d t+$ $\frac{2 c(\tau)}{c_{1}} \int_{\gamma(b-a)}^{\tau-\gamma(b-a)} E_{c}(t) d t$, with $c(\tau)=\frac{c_{1}}{2(\tau-2 \gamma(b-a))}$. The second inequality is obtained similarly with $F(z)=$ $\int_{\gamma(a-z)}^{\tau-\gamma(a-z)} x^{\top} \mathcal{L} x d t$

Theorem 12. Consider the BCS defined by Theorem 4 with $r(t)=0$, for all $t \geq 0$. The finite dimensional boundary controller of Definition 6 exponentially stabilizes the BCS, provided Assumption 10 is satisfied.

Proof. Let $\sigma>0$ be such that $S_{c} \geq \sigma I$. The time derivative of the total energy satisfies

$$
\begin{aligned}
\dot{\tilde{E}}= & -v^{\top} Q_{c} R_{c} Q_{c} v-u_{c}^{\top} S_{c} u_{c} \\
\leq & -v^{\top} Q_{c} R_{c} Q_{c} v-\sigma u_{c}^{\top} u_{c}, \quad \text { since } S_{c} \geq \sigma I \\
= & -v^{\top} Q_{c} R_{c} Q_{c} v-\sigma \epsilon_{1} u_{c}^{\top} u_{c}-\sigma \epsilon_{2} u_{c}^{\top} u_{c} \\
= & -v^{\top} Q_{c} R_{c} Q_{c} v-\sigma \epsilon_{1}\left\|u_{c}\right\|^{2}-\sigma \epsilon_{2}\left(\|y\|^{2}+\|u\|^{2}\right)+ \\
& \sigma \epsilon_{2}\|u\|^{2}
\end{aligned}
$$

with $\epsilon_{1}+\epsilon_{2}=1$ and where we have used that $u_{c}=-y$. Using Assumption 10 we have

$$
\begin{aligned}
\dot{\tilde{E}} \leq-v^{\top} Q_{c} R_{c} Q_{c} v & \\
& -\sigma \epsilon_{1}\left\|u_{c}\right\|^{2}-\sigma \epsilon_{2} \epsilon\|\mathcal{L} x(t, b)\|^{2}+\sigma \epsilon_{2}\left\|y_{c}\right\|^{2} .
\end{aligned}
$$

Integrating this equation on $t \in[0, \tau]$ we have

$$
\begin{aligned}
& \tilde{E}(\tau)-\tilde{E}(0) \leq-\int_{0}^{\tau} v^{\top}(t) Q_{c} R_{c} Q_{c} v(t) d t \\
& +\int_{0}^{\tau}-\sigma \epsilon_{1}\left\|u_{c}(t)\right\|^{2}-\sigma \epsilon_{2} \epsilon\|\mathcal{L} x(t, b)\|^{2}+\sigma \epsilon_{2}\left\|y_{c}(t)\right\|^{2} d t
\end{aligned}
$$

Next choose $\tau$ sufficiently large such that Lemmas 8 and 11 hold. Using the latter lemma we have

$$
\begin{aligned}
& \tilde{E}(\tau)-\tilde{E}(0) \leq-\int_{0}^{\tau} v^{\top} Q_{c} R_{c} Q_{c} v+\sigma \epsilon_{1}\left\|u_{c}\right\|^{2} d t \\
& +\frac{\sigma \epsilon_{2} \epsilon}{c(\tau)}\left(\frac{2 c(\tau)}{c_{1}} \int_{0}^{\tau} E_{c}(t) d t-\tilde{E}(\tau)\right)+\sigma \epsilon_{2} \int_{0}^{\tau}\left\|y_{c}\right\|^{2} d t .
\end{aligned}
$$

Grouping terms we have that

$$
\begin{aligned}
& \tilde{E}(\tau)\left(1+\frac{\sigma \epsilon_{2} \epsilon}{c(\tau)}\right)-\tilde{E}(0) \leq \\
& -\int_{0}^{\tau} v(t)^{\top} Q_{c} R_{c} Q_{c} v(t) d t-\sigma \epsilon_{1} \int_{0}^{\tau}\left\|u_{c}(t)\right\|^{2} d t \\
& \quad+\sigma \epsilon_{2}\left(\int_{0}^{\tau} \frac{2 \epsilon}{c_{1}} E_{c}(t)+\left\|y_{c}(t)\right\|^{2} d t\right) .
\end{aligned}
$$

Using Lemma 9 with $\delta_{1}=\frac{2 \epsilon}{c_{1}}$ we have

$$
\begin{aligned}
& \tilde{E}(\tau)\left(1+\frac{\sigma \epsilon_{2} \epsilon}{c(\tau)}\right)-\tilde{E}(0) \leq-\int_{0}^{\tau} v(t)^{\top} Q_{c} R_{c} Q_{c} v(t) d t \\
& +\sigma \epsilon_{2} \delta_{2} \int_{0}^{\tau} E_{c}(t) d t+\sigma\left(\epsilon_{2} \delta_{2}-\epsilon_{1}\right) \int_{0}^{\tau}\left\|u_{c}(t)\right\|^{2} d t
\end{aligned}
$$

Now, using Lemma 8 we obtain

$$
\begin{aligned}
& \tilde{E}(\tau)\left(1+\frac{\sigma \epsilon_{2} \epsilon}{c(\tau)}\right)-\tilde{E}(0) \leq \\
& \left(\sigma \epsilon_{2} \delta_{2} \xi_{1}-1\right) \int_{0}^{\tau} v(t)^{\top} Q_{c} R_{c} Q_{c} v(t) d t+ \\
& \sigma\left(\epsilon_{2} \delta_{2}\left(1+\xi_{2}\right)-\epsilon_{1}\right) \int_{0}^{\tau}\left\|u_{c}(t)\right\|^{2} d t
\end{aligned}
$$


Since $\epsilon_{2}$ may be chosen to be arbitrarily small, i.e, $\epsilon_{2} \ll 1$ and since $\epsilon_{1}=1-\epsilon_{2}$, we finally have that $\tilde{E}(\tau) \leq c_{2} \tilde{E}(0)$ with $c_{2}=\frac{1}{\left(1+\frac{\sigma \epsilon_{2} \epsilon}{c(\tau)}\right)}<1$ which proves the theorem.

\section{DNA-MANIPULATION PROCESS}

In this section we focus on the stability analysis of a controlled nanotweezer used for DNA manipulation (Boudaoud et al., 2012). The tweezers is presented in Figure 1.

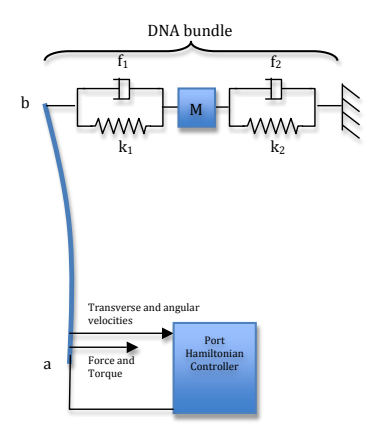

Fig. 1. DNA manipulation with $\mathrm{PH}$ control

The trapped DNA bundle has been approximated in Ramirez and Le Gorrec (2013a) by a mass-spring-damper system attached at the tip of the tweezer. Here we consider a more accurate model made up by two spring-damper systems interconnected to a moving load. The arm is actuated on one side (by applying a force and a torque at the point $a$ ) by using electrostatic forces generated by a comb drive actuator, the beam being clamped to the moving shuttle. We also assume that it is only possible to measure the transversal and angular velocities at the point $a$. The total system, may be divided into three subsystems: the flexible arm, the DNA-bundle at the tip of the gripper and the port Hamiltonian controller. The flexible arm is modelled as a Timoshenko beam (infinite dimensional system) while the DNA-bundle is modelled as a finite dimensional mechanical system. The subsystems are interconnected through their boundary power conjugated port variables. .

\subsection{The Timoshenko beam}

The Timoshenko beam has been widely studied as a distributed parameter port Hamiltonian system (Macchelli and Melchiorri, 2004) and as BCS (Le Gorrec et al., 2005). The exponential stability of the system has been proved for static boundary feedback (Villegas, 2007; Villegas et al., 2009). The BCS is defined as

$$
\frac{\partial}{\partial t}\left[\begin{array}{l}
x_{1} \\
x_{2} \\
x_{3} \\
x_{4}
\end{array}\right]=\underbrace{\left[\begin{array}{llll}
0 & 1 & 0 & 0 \\
1 & 0 & 0 & 0 \\
0 & 0 & 0 & 1 \\
0 & 0 & 1 & 0
\end{array}\right]}_{P_{1}} \frac{\partial}{\partial z}\left[\begin{array}{c}
K x_{1} \\
\frac{1}{\rho} x_{2} \\
E I x_{3} \\
\frac{1}{I_{\rho}} x_{4}
\end{array}\right]+\underbrace{\left[\begin{array}{cccc}
0 & 0 & 0 & -1 \\
0 & 0 & 0 & 0 \\
0 & 0 & 0 & 0 \\
1 & 0 & 0 & 0
\end{array}\right]}_{P_{0}}\left[\begin{array}{c}
K x_{1} \\
\frac{1}{-} x_{2} \\
\frac{\rho}{E I x_{3}} \\
\frac{1}{I_{\rho}} x_{4}
\end{array}\right]
$$

where the following state (energy) variables have been defined: $x_{1}=\frac{\partial w}{\partial z}(z, t)-\phi(z, t)$ the shear displacement, $x_{2}=\rho(z) \frac{\partial w}{\partial t}(z, t)$ the transverse momentum distribution, $x_{3}=\frac{\partial \phi}{\partial z}(z, t)$ the angular displacement, and $x_{4}=$
$I_{\rho} \frac{\partial \phi}{\partial t}(z, t)$ the angular momentum distribution, for $z \in$ $(a, b), t \geq 0$, where $w(t, z)$ is the transverse displacement of the beam and $\phi(t, z)$ is the rotation angle of a filament of the beam. The coefficients $\rho(z), I_{\rho}(z), E(z), I(z)$ and $K(z)$ are the mass per unit length, the rotary moment of inertia of a cross section, Young's modulus of elasticity, the moment of inertia of a cross section, and the shear modulus respectively. The matrices $P_{1}$ and $P_{0}$ defines the skewsymmetric differential operator of order 1 acting on the state space $X=L_{2}\left(a, b, \mathbb{R}^{4}\right), \mathcal{J}=P_{1} \frac{\partial}{\partial z}+P_{0}$. The energy of the beam is expressed in terms of the energy variables, $E=\frac{1}{2} \int_{a}^{b}\left(K x_{1}^{2}+\frac{1}{\rho} x_{2}^{2}+E I x_{3}^{2}+\frac{1}{I_{\rho}} x_{4}^{2}\right) d z=\frac{1}{2}\|x\|_{\mathcal{L}}^{2}$. The boundary port variables are obtained by using integration by parts and factorization in order to define an extended Dirac structure including the boundary (Le Gorrec et al., 2005). They also can be directly parametrized from $P_{1}$ (Le Gorrec et al., 2005; Villegas, 2007) leading to:

$$
\left[\begin{array}{c}
f_{\partial, \mathcal{L} x} \\
e_{\partial, \mathcal{L} x}
\end{array}\right]=\left[\begin{array}{c}
\left(\rho^{-1} x_{2}\right)(b)-\left(\rho^{-1} x_{2}\right)(a) \\
\left(K x_{1}\right)(b)-\left(K x_{1}\right)(a) \\
\left(I_{\rho}^{-1} x_{4}\right)(b)-\left(I_{\rho}^{-1} x_{4}\right)(a) \\
\left(E I x_{3}\right)(b)-\left(E x_{3}\right)(a) \\
\left(\rho^{-1} x_{2}\right)(b)+\left(\rho^{-1} x_{2}\right)(a) \\
\left(K x_{1}\right)(b)+\left(K x_{1}\right)(a) \\
\left(I_{\rho}^{-1} x_{4}\right)(b)+\left(I_{\rho}^{-1} x_{4}\right)(a) \\
\left(E I x_{3}\right)(b)+\left(E I x_{3}\right)(a)
\end{array}\right]
$$

The control objective is to control the translational and angular position of the DNA-bundle. The physical ports are given by the translational force acting at the base of the beam (input), and the translational velocity at the base of the beam (output). All physical ports are hence located on the point $a$ of the beam and directly associated with the dynamic of the suspension mechanism and/or base of the beam. In order to achieve that the input and output variables of the flexible arm coincide with the physical ones we define the following input and outputs for the beam:

$$
u=[v(b) \omega(b) F(a) T(a)], \quad y=[F(b) T(b)-v(a)-\omega(a)],
$$

which is achieved by defining $u=W\left[\begin{array}{c}f_{\partial, \mathcal{L} x} \\ e_{\partial, \mathcal{L} x}\end{array}\right], y=$ $\tilde{W}\left[\begin{array}{l}f_{\partial, \mathcal{L} x} \\ e_{\partial, \mathcal{L} x}\end{array}\right]$ where

$$
W=\left[\begin{array}{cccccccc}
1 & 0 & 0 & 0 & 0 & 1 & 0 & 0 \\
0 & 0 & 1 & 0 & 0 & 0 & 0 & 1 \\
0 & -1 & 0 & 0 & 1 & 0 & 0 & 0 \\
0 & 0 & 0 & -1 & 0 & 0 & 1 & 0
\end{array}\right], \tilde{W}=\left[\begin{array}{cccccccc}
0 & 1 & 0 & 0 & 1 & 0 & 0 & 0 \\
0 & 0 & 0 & 1 & 0 & 0 & 1 & 0 \\
1 & 0 & 0 & 0 & 0 & -1 & 0 & 0 \\
0 & 0 & 1 & 0 & 0 & 0 & 0 & -1
\end{array}\right]
$$

It can by shown that with this choice of input and output the system (25) defines a an abstract boundary control system. Furthermore $\mathcal{A} x=P_{1}(\partial / \partial z)(\mathcal{L} x)+P_{0} \mathcal{L} x$ with domain $D(\mathcal{A})=\left\{\mathcal{L} x \in H^{1}\left(a, b ; \mathbb{R}^{n}\right) \mid\left[\begin{array}{c}f_{\partial, \mathcal{L} x} \\ e_{\partial, \mathcal{L} x}\end{array}\right] \in \operatorname{ker} W\right\}$ generates a contraction semigroup on $X$ and the energy balance equation is defined as: $\frac{d E}{d t}=u^{T} y$

Remark 13. In our application case the Timoshenko beam is fully actuated and from the full rankness of $\left[W^{T} \widetilde{W}^{T}\right]^{T}$ and $P_{1}$ and in the definition of $u$ and $y$ from (2), the associated BCS satisfies Assumption 10.

\subsection{DNA-bundle model}

The DNA-bundle is represented by the simple springdamper + load + spring-damper system of Figure 2 and thus admits a port Hamiltonian system representation. In Figure $2, k_{1}, k_{2}, f_{1}, f_{2}$ represent the positive constants of the springs and the viscous dampers respectively, $M$ is the mass of the load, $x_{c 1}, x_{c 2}$ the relative positions. Let 


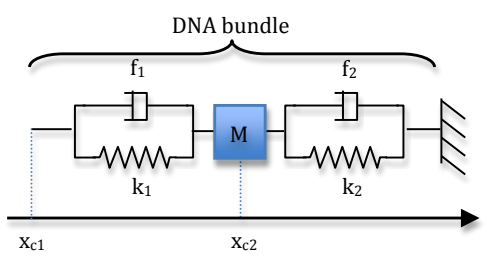

Fig. 2. DNA model

us denote with the sub-index $\mathrm{b}$ the system representing the DNA-bundle. Then we can write by using $v_{b}=\left(x_{c 2}-\right.$ $\left.x_{c 1}, x_{c 2}, M \dot{x}_{c 2}\right)^{T}, u_{b}=[F(b) T(b)]^{T}$ and $y_{b}=[v(b) \omega(b)]^{T}$ :

$$
\dot{v}_{b}=\left(J_{b}-R_{b}\right) \frac{d E_{b}}{d v_{b}}+g_{b} u_{b}, \quad y_{b}=g_{b}^{T} \frac{d E_{b}}{d v_{b}}+S_{b} u_{b}
$$

with $E_{b}$ the energy of the system (sum of the kinetic and potential energies): $E_{b}\left(x_{c 2}-x_{c 1}, x_{c 2}, M \dot{x}_{c 2}\right)=\frac{k_{1}}{2}\left(x_{c 2}-\right.$ $\left.x_{c 1}\right)^{2}+\frac{k_{2}}{2} x_{c 2}^{2}+\frac{1}{2 M}\left(M \dot{x}_{c 2}\right)^{2}$ and $J_{b}=\left[\begin{array}{ccc}0 & 0 & 0 \\ 0 & 0 & 1 \\ 0 & -1 & 0\end{array}\right], \quad R_{b}=$ $\left[\begin{array}{ccc}\frac{k_{1}}{f_{1}} & 0 & 0 \\ 0 & 0 & 0 \\ 0 & 0 & f_{2}\end{array}\right], g_{b}^{T}=\left[\begin{array}{ccc}\frac{1}{f_{1}} & 0 & 1 \\ 0 & 0 & 0\end{array}\right], S_{b}=\left[\begin{array}{cc}\frac{1}{f_{1}} & 0 \\ 0 & \frac{1}{f_{\theta}}\end{array}\right]$, where $f_{\theta}$ is the rotational damping constant at the interconnection point. Note that this system is exponentially stable.

\section{THE CONTROLLER}

The controller is set at the base of the flexible arm, and is free to design. Hence the controller should be selected such that the conditions in Theorem 12 are satisfied. To this end we chose the "a-part" of the controller as

$$
\dot{v}_{a}=\left(J_{a}-R_{a}\right) \frac{d E_{a}}{d v_{a}}+g_{a} u_{a}, \quad y_{a}=g_{a}^{\top} \frac{d E_{a}}{d v_{a}}+S_{a} u_{a},
$$

where $v_{a}=\left[q_{a_{1}}, q_{a_{2}}, p_{a_{1}}, p_{a_{2}}\right]^{\top}, q_{a_{1}}, q_{a_{2}}$ are the generalized coordinates, $J_{a}, R_{a}, S_{a} \in M_{4}(\mathbb{R}), J_{a}=\left[\begin{array}{cc}0 & I \\ -I & 0\end{array}\right]$, $R_{a}=\left[\begin{array}{cc}R_{a 1} & 0 \\ 0 & R_{a 2}\end{array}\right], S_{a}=\left[\begin{array}{cc}S_{a 1} & 0 \\ 0 & S_{a 2}\end{array}\right], g_{a}=\left[\begin{array}{l}0 \\ I\end{array}\right]$ with $R_{a i}=$ $\left[\begin{array}{cc}r_{a_{i 1}} & 0 \\ 0 & r_{a_{i 2}}\end{array}\right] \in M_{2}(\mathbb{R}), r_{a_{i 1}}, r_{a_{i 2}}>0 \in \mathbb{R}, S_{a i}=$ $\left[\begin{array}{cc}S_{a_{i 1}} & 0 \\ 0 & s_{a_{i 2}}\end{array}\right] \in M_{2}(\mathbb{R}), s_{a_{i 1}}, s_{a_{i 2}}>0 \in \mathbb{R}, i=1,2$. The Hamiltonian of the system is given by the virtual energy: $E_{a}=\frac{1}{2}\left(k_{a_{1}} q_{a_{1}}^{2}+k_{a_{2}} q_{a_{2}}^{2}\right)+\frac{1}{2}\left(\frac{p_{a_{1}}^{2}}{m_{a}}+\frac{p_{a_{2}}^{2}}{m_{I_{a}}}\right)$ where $k_{a_{1}}, k_{a_{2}}, m_{a}, m_{I_{a}}$ are (positive) tuning parameters. The inputs $u_{a}=\left[u_{a_{1}}, u_{a_{2}}\right]^{\top} \in \mathbb{R}^{2}$ may be identified with the boundary variables of the beam at the point $a, u_{a}=$ $[-v(a),-\omega(a)]^{\top}$. The outputs correspond to input force and torque of the beam at point $a, y_{a}=[F(a), T(a)]^{\top}$. The finite dimensional PHS hence corresponds to an input strictly passive, exponentially stable system. The complete finite dimensional PHS may hence be written by combining the PHS representing the suspension mechanism and DNA-bundle,

$$
\begin{aligned}
\dot{v} & =\left[\begin{array}{c|c}
J_{b}-R_{b} & 0 \\
\hline 0 & J_{a}-R_{a}
\end{array}\right] \frac{d E_{c}}{d v}+\left[\begin{array}{l}
g_{b} \\
g_{a}
\end{array}\right] u_{c} \\
y_{c} & =\left[\begin{array}{ll}
g_{b}^{\top} & g_{a}^{\top}
\end{array}\right] \frac{d E_{c}}{d v}+\left[\begin{array}{c|c}
S_{a} & 0 \\
\hline 0 & S_{b}
\end{array}\right] u_{c}
\end{aligned}
$$

The finite dimensional PHS is a strictly passive and exponentially stable system with state $v=\left[v_{a}, v_{b}\right]^{\top}$, Hamiltonian (storage) function $E_{c}=E_{a}+E_{b}$, input $u_{c}=\left[u_{a}, u_{b}\right]^{\top}$, supply rate $w=u_{c} y_{c}$ and quadratic dissipation rate $s={\frac{d E_{a}}{d v_{a}}}^{\top} R_{a} \frac{d E_{a}}{d v_{a}}+\frac{d E_{b}}{d v_{b}}{ }^{\top} R_{b} \frac{d E_{b}}{d v_{b}}+u_{a}^{\top} S_{a} u_{a}+$ $u_{b}^{\top} S_{b} u_{b}$. Hence the microgripper i.e., the interconnection of the flexible arm, DNA-bundle and controller is an exponentially stable system by Theorem 12 .

\section{CONCLUSION}

It has been shown that a large class of boundary control systems are exponentially stable if they are interconnected in a power preserving manner with an exponentially stable input strictly passive linear finite dimensional system. The result follows since the finite dimensional system dissipates the energy flowing through the boundaries of the infinite dimensional system. The assumptions made on the infinite dimensional systems are that it is port-Hamiltonian and that the norm of energy flowing through one boundary is always less than sum of the norms of the input and the output. The assumptions on the finite dimensional system is that it is exponentially stable and input strictly passive. The result has been illustrated on the physical example of a DNA-manipulation process. Future work will deal with the relaxation of the input strictly passive condition of the finite dimensional controller.

\section{ACKNOWLEDGEMENTS}

The authors want to thanks Björn Augner and Birgit Jacob for their careful reading of the first draft version of this paper, which turned out to be full of typo's.

\section{REFERENCES}

Boudaoud, M., Haddab, Y., and Le Gorrec, Y. (2012). Modeling and optimal force control of a nonlinear electrostatic microgripper. Mechatronics, IEEE/ASME Transactions on, $\mathrm{PP}(99), 1-10$. doi: 10.1109/TMECH.2012.2197216.

Brogliato, B., Lozano, R., Maschke, B., and Egeland, O. (2007). Dissipative Systems Analysis and Control. Communications and Control Engineering Series. Springer Verlag, London, 2nd edition edition.

Curtain, R. and Zwart, H. (1995). An introduction to infinitedimensional linear systems theory. Texts in applied mathematics. Springer-Verlag, New York, USA.

Jacob, B. and Zwart, H. (2012). Linear Port-Hamiltonian Systems on Infinite-dimensional Spaces, volume 223 of Operator Theory: Advances and Applications. Birkhäuser, Basel, Switzerland.

Le Gorrec, Y., Zwart, H., and Maschke, B. (2005). Dirac structures and boundary control systems associated with skew-symmetric differential operators. SIAM Journal on Control and Optimization, 44(5), 18641892 .

Macchelli, A. (2012). Boundary energy shaping of linear distributed port-Hamiltonian systems. In Proceedings of the 4th IFAC workshop on Lagrangian and Hamiltonian methods for non-linear control. Bertinoro, Italy.

Macchelli, A. and Melchiorri, C. (2004). Modeling and control of the Timoshenko beam. the distributed port Hamiltonian approach. SIAM Journal on Control and Optimization, 43(2), 743-767.

Ramirez, H. and Le Gorrec, Y. (2013a). Boundary port hamiltonian control of a class of nanotweezers. In Proceedings of the European Control Conference 2013. Zurich, Switzerland.

Ramirez, H. and Le Gorrec, Y. (2013b). Exponential stability of a class of PDE's with dynamic boundary control. In Proceedings of the 2013 American Control Conference. Washington, USA.

van der Schaft, A.J. (2000). L2-Gain and Passivity Techniques in Nonlinear Control. Springer-Verlag, New York, USA, 2st edition.

Villegas, J., Zwart, H., Le Gorrec, Y., and Maschke, B. (2009). Exponential stability of a class of boundary control systems. IEEE Transactions on Automatic Control, 54, 142-147.

Villegas, J., Zwart, H., Le Gorrec, Y., Maschke, B., and van der Schaft, A. (2005). Stability and stabilization of a class of boundary control systems. In Decision and Control, 2005 and 2005 European Control Conference. CDC-ECC' '05. 44th IEEE Conference on, 3850-3855.

Villegas, J.A. (2007). A port-Hamiltonian Approach to Distributed Parameter Systems. Ph.D. thesis, Universiteit Twente. 